\title{
Heparin-induced Thrombocytopenia Type II after Free Flap Operation
}

\author{
Jiwoong Baek, Jung Hyun Park, In-Ho Cha, Hyung Jun Kim \\ Department of Oral and Maxillofacial Surgery, Yonsei University College of Dentistry
}

\begin{abstract}
After radical excision of a tumor in the maxillofacial area, functional and esthetic reconstruction is needed, including flap surgery. Among the many etiologies of flap failure, venous thrombosis is one of the most frequent. Heparin is used routinely in the effort to avoid development of venous thrombosis. In rare cases, heparin-induced thrombocytopenia (HIT) type II occurs due to exposure to heparin. Heparin attached to platelet factor 4 forms a PF4/heparin-immunoglobulin $G$ immune complex on platelet surfaces. This complex activates platelets, which leads to multiple coagulation in venous and arterial blood. We report here on a rare occurrence of HIT type II following fibula free flap surgery.
\end{abstract}

Key words: HIT type II, Heparin-induced thrombocytopenia, Free tissue flaps, Heparin

\section{Introduction}

Heparin-induced thrombocytopenia (HIT) type II is defined as the formation of systemic thrombus accompanied by a more than $50 \%$ reduction of platelet count, with no other explanation than exposure to heparin[1].

HIT is important in reconstruction due to the inevitable use of heparin in free flap surgery[2]. Thrombocytopenia due to HIT type II influences flap survival. Even small amounts of heparin, such as from irrigation or a catheter coating, can trigger HIT[3]. During a low-platelet period, vascular conditions can give rise to a thrombus, which can cause organ failure and finally death[4].

There are two types of HIT. Type I HIT is momentary, occurring approximately four days after exposure to heparin. Minor thrombocytopenia springs up and is not associated with immunoglobulins. Type II HIT is mediated by immunoglobulin $G$ ( $\operatorname{IgG}$ ) and manifests as a critical diminution in platelet count 4 to 14 days after exposure to heparin. Potentially fatal complications require immediate intervention[5]. However, this is unusual and rarely occurs.

\section{Case Report}

A 47-year-old man came to Department of Oral and Maxillofacial Surgery, Yonsei University College of Dentistry for evaluation and treatment of squamous cell carcinoma in the right mandibular retromolar area. The patient had no specific medical history, and baseline international normalized ratio (INR) blood test was 1.06 , and activated partial thromboplastin time (aPTT) was 29.6. Platelet count was 242,000, and glutamic oxaloacetic transaminase (GOT)/

RECEIVED August 9, 2013, REVISED September 7, 2013, ACCEPTED November 26, 2013

Correspondence to Hyung Jun Kim

Department of Oral and Maxillofacial Surgery, Yonsei University College of Dentistry

50 Yonsei-ro, Seodaemun-gu, Seoul 120-752, Korea

Tel: 82-2-2228-3132, Fax: 82-2-2227-8022, E-mail: kimoms@yuhs.ac

(C) This is an open access article distributed under the terms of the Creative Commons Attribution Non-Commercial License (http://creativecommons. org/licenses/ by-nc/3.0) which permits unrestricted non-commercial use, distribution, and reproduction in any medium, provided the original work is properly cited. 
glutamic pyruvic transaminase (GPT) was 16/11, both within normal range. He had received treatment in a local clinic with repetitive curettage on the right third molar. Finally, the dentist performed a biopsy, from which squamous cell carcinoma was determined.

Following clinical evaluation, computed tomography (CT), magnetic resonance imaging (MRI) (Fig. 1), and positron emission tomography-CT (PET-CT), CT lower extremity angio (Fig. 2) examinations were performed. The center of the lesion was located in the right mandibular body, involving the right masseter and medial and lateral pterygoid muscles. The lesion extended to the right mandibular trigone and mandibular cortex, medullary portion. The right neck showed reactive lymph nodes at levels I and II. An operation was planned, and wide excision of the lesion, modified radical neck dissection on the right neck, supraomohyoid neck dissection on the left neck, and reconstruction of soft and hard tissue defect with a fibula free flap was performed. After the operation, the patient went into the intensive care unit (ICU). Subcutaneous injection of 5,000 units of un-fractioned heparin was administered as an anti-thrombotic and compression stockings were used on both legs. Daily routine lab tests were performed, including platelet count. Two days after the operation, the patient was moved from the ICU to the general ward. Seven days after the operation, the patient showed sudden hypooxygenation and dyspnea,

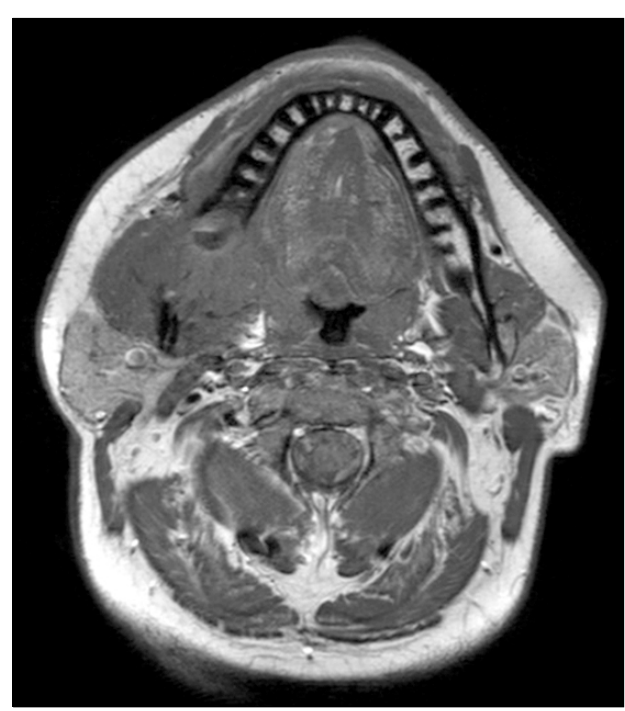

Fig. 1. Preoperative magnetic resonance imaging (T2) view. Right mandibular lesion extends to the masseter, medial pterygoid, and mouth floor. and thus returned to the ICU.

Eight days after the operation, congestion was seen on the skin portion of the free flap area. A Doppler test was performed immediately and blood flow through vessels was maintained. Daily lab tests showed a slight reduction of the platelet count. Eleven days after the operation, the platelet count fell to $26,000 / \mu \mathrm{L}$ (Fig. 3). For evaluation of systemic thrombosis, an ultrasonography test was performed on the legs and arms, and a chest CT was taken, which showed pulmonary thromboembolism and partial pulmonary infarction. The 4 T's test indicated a more than 50\% reduction in platelet count within 5 to 10 days after the operation and thrombus formation with no other remark-

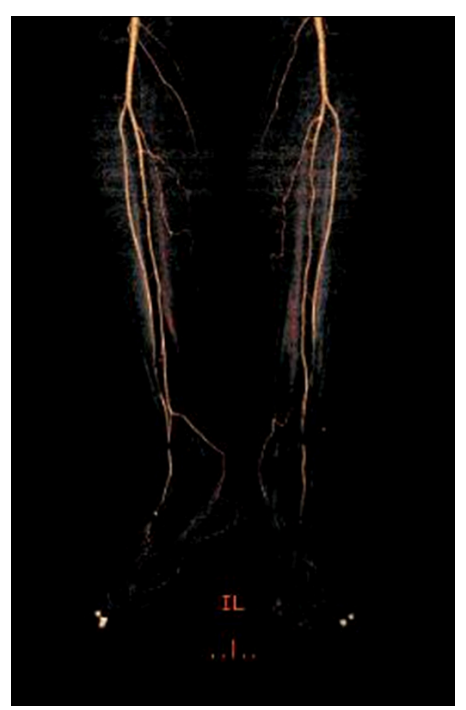

Fig. 2. Preoperative computed tomography lower extremity angiography. Both hyperplastic posterior tibial arteries were seen, and no other remarkable lesion was observed.

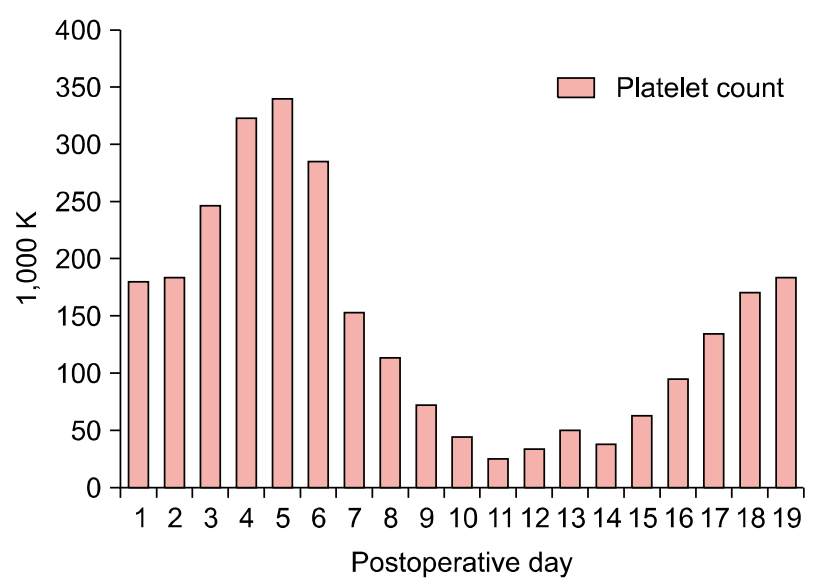

Fig. 3. Evaluation of postoperative platelet count (19 days). On postoperative day 11 , platelet count fell to $26,000 / \mu \mathrm{L}$. 
able reason. Evaluation of the 4 T's test was performed, yielding a score of 8 points, the highest score. HIT type II was strongly suspected.

A hematologist was consulted immediately. Clinically, HIT type II was diagnosed. Heparin in any amount whatsoever was discontinued. Because the patient had no specific renal insufficiency, and thromboses were found, we could select argatroban or lepirudin or danapariod based on American College of Chest Physicians Evidence-Based Clinical Practice Guidelines (currently, 2012 American CHEST physicians' guidelines are the newest[6]).

Intravenous argatroban (Novastan; Mitsubishi Tanabe Pharma Korea, Seoul, Korea) $10 \mathrm{mg}$ with infusion of 100 $\mathrm{mL}$ normal saline was started, and dose was regulated to target $\mathrm{INR}=2.5$ and $\mathrm{aPTT}=50$ to 55 seconds using an infusion pump, which was approximately two times the patient's baseline. Monitoring of aPTT was performed at 2-hours intervals.

Fourteen days after the operation, the platelet count began to increase. Argatroban was maintained for 25 days, followed by coumadization. On discharge, the patient's platelet count was $366,000 / \mu \mathrm{L}$, and INR was 2.58 . The skin portion of the flap was partially dead and was therefore removed. The muscular and bone portion of the flap finally survived.

\section{Discussion}

There are many causes of flap failure, including thrombosis. Jeon et al.[7] reported a free flap survival rate of $98.8 \%$ among 164 cases. Total failure occurred in two cases, other complications included partial flap necrosis, delayed healing of donor site, infection, and fistula formation.

Heparin is used routinely for maintenance of blood flow and prevention of thrombosis. Its most common complication is bleeding. HIT type II is a rare disease compared to other complications, exposure of heparin constituting its predisposing factor. Although there is no bleeding tendency in HIT type 2, there is usually systemic coagulation.

Smythe et al.[8] reported that the incidence of HIT type II is $0.2 \%$. Warkentin et al.[9] reported that the incidence may increase to $1 \%$ in the context of major surgery. After the operation, damaged endothelial cells cause platelets and platelet factor 4 (PF4) to become more active, increasing the likelihood of HIT. Use of low molecular weight heparin (LMWH) decreases the incidence of HIT.

HIT type II is associated with an immune reaction related to IgG, which binds to heparin and forms negatively charged antibodies against PF4. The antibodies bind with negatively charged PF4, forming a complex involved in platelet activation, coagulation, and endothelial activity. Platelet factor is accelerated by cross-linking of heparin-IgG/PF4 complex, which can lead to strong coagulation. Reduction of platelet count occurs 4 to 14 days after exposure to heparin, but can occur within as little as 24 hours[10].

HIT type II may cause three critical complications: pulmonary embolism, adrenal failure, and acute anaphylactoid reaction. Venous thrombosis occurs in $25 \%$ to $75 \%$ of HIT type II patients, and approximately one fourth of them have arterial thrombosis (4:1 venous to arterial ratio). Approximately $25 \%$ of HIT type II patients experience adrenal failure and adrenal vein thrombosis may develop into adrenal hemorrhagic necrosis. Development of acute renal crisis can occur when adrenal glands are bilaterally dead.

Manifestations of anaphylactoid reaction include acute hypertension, dyspnea, fever, chills, chest pain, and tachycardia, with severe cases resulting in respiratory or cardiogenic arrest. In our case, the patient showed dyspnea seven days after the operation and re-entered the ICU.

Because of these risk factors, Warkentin[10] recommended that HIT type II patients should receive care in the ICU. Based on reported cases, approximately $4 \%$ to $5 \%$ of HIT type II patients are at risk of death[4].

For prevention of HIT, unfractioned heparin (UFH) can be replaced by LMWH, which generally shows lower incidence of HIT than UFH. However, the risk of HIT remains with LMWH, which is more costly.

Although HIT is clearly dangerous, there is no viable replacement for heparin, drugs such as direct thrombin inhibitor (DTI) notwithstanding. Heparin, the most valuable anti-thrombotic drug, has the advantages of not going over liver and kidney and its anti-thrombotic effect can be easily reversed by protamine. In addition, DTI agents such as argatroban and lepirudin should be regulated using bleeding markers such as partial thromboplastin time value, complicated and laborious work, which can easily fail. Most patients who receive heparin therapy are free of HIT.

For evaluation of flap survival, the clinician must perform an inspection for several signs. Detection of congestion 
and swelling, which is sometimes fatal to flap survival, is dependent on repeated color inspection, pressure test for perfusion, and ultrasonic Doppler test. If thrombosis accompanied by an abrupt decrease in platelet count is observed, the clinician should consider the possibility of HIT type II, whose conclusive diagnosis requires recovery of platelet count after discontinuing heparin. Other diagnostic methods for HIT type II are platelet serotonin-release assay, heparin induced platelet activation test, IgG-specific PF4-dependent enzyme immunoassays, and polyspecific enzyme immunoassays[11]. As these lab tests take considerable time, the clinician often reaches a diagnosis based on the clinical situation. In this case, these lab tests were not performed due to lack of assay facilities.

It is important that clinicians understand the following: when thrombosis is found, its cause must be determined. Virchow's triad is useful in this respect. Virchow proposed that three elements can lead to development of thrombosis: stasis, changes in the vessel wall, and changes in the blood. If there are no other reasons for thrombosis and platelet count starts to decrease, the 4 T's scoring system can be utilized[12]. Elements include 1) platelet fall, 2) timing of platelet fall, 3) thrombosis or other clinical sequelae, and 4) other causes of thrombocytopenia not evident. Each element counts for 0,1 , or 2 points. The sum of the points indicates the risk value for HIT, with low risk being 0 to 3 points, intermediate risk 4 to 5 points, and high risk 6 to 8 points.

If HIT type II is presumed, immediate consultation to a hematologist is necessary. Prognosis of HIT type II is dependent on early detection and early treatment. Five steps recommended for handling a diagnosis of HIT type II include stopping all heparin medication, consultation to a hematologist, use of another antithrombotic agent, imaging study for thrombosis, and study of antibodies[12].

We used argatroban instead of heparin. After discontinuing heparin, the platelet count starts to increase. In treatment of HIT, additional heparin injection is not allowed, as this can cause massive coagulation followed by systemic thrombosis

DeBois et al.[13] recommended at least two platelet counts per week when using heparin. American CHEST physicians' guidelines[6] recommend one platelet count per two days. As the operation can increase the incidence of HIT type II, we propose a daily platelet count check two weeks post-operatively. HIT type II usually starts 4 to 14 days after the operation.

HIT type II is a rare disease related to heparin exposure, which leads to coagulation disorder. Local or systemic coagulation may cause flap failure and other fatal complications. When flap congestion accompanied by an abrupt decrease in platelet count occurs for no particular reason, the clinician should consider HIT type II. Early diagnosis and treatment is essential.

\section{References}

1. Warkentin TE, Roberts RS, Hirsh J, Kelton JG. An improved definition of immune heparin-induced thrombocytopenia in postoperative orthopedic patients. Arch Intern Med 2003;163: 2518-24.

2. Tremblay DM, Harris PG, Gagnon AR, Cordoba C, Brutus JP, Nikolis A. Heparin-induced thrombocytopenia syndrome as a cause of flap failure: a report of two cases. J Plast Reconstr Aesthet Surg 2008;61:78-83.

3. Laster J, Silver D. Heparin-coated catheters and heparin-induced thrombocytopenia. J Vasc Surg 1988;7:667-72.

4. Warkentin TE, Aird WC, Rand JH. Platelet-endothelial interactions: sepsis, HIT, and antiphospholipid syndrome. Hematology Am Soc Hematol Educ Program 2003:497-519.

5. Spinler SA, Dager W. Overview of heparin-induced thrombocytopenia. Am J Health Syst Pharm 2003;60 Suppl 5:S5-11.

6. Linkins LA, Dans AL, Moores LK, et al; American College of Chest Physicians. Treatment and prevention of heparin-induced thrombocytopenia: Antithrombotic Therapy and Prevention of Thrombosis, 9th ed: American College of Chest Physicians Evidence-Based Clinical Practice Guidelines. Chest 2012;141:e495S-530S.

7. Jeon JH, Park SW, Jo SH, Park JY, Lee JH, Choi SW. Analysis of outcome and complications in 164 cases of free flap reconstructions: experience of a National Cancer Center. J Korean Assoc Maxillofac Plast Reconstr Surg 2011;33:478-82.

8. Smythe MA, Koerber JM, Mattson JC. The incidence of recognized heparin-induced thrombocytopenia in a large, tertiary care teaching hospital. Chest 2007;131:1644-9.

9. Warkentin TE, Levine MN, Hirsh J, et al. Heparin-induced thrombocytopenia in patients treated with low-molecular-weight heparin or unfractionated heparin. N Engl J Med 1995;332:1330-5.

10. Warkentin TE. Heparin-induced thrombocytopenia in critically ill patients. Crit Care Clin 2011;27:805-23, v.

11. Warkentin TE. How I diagnose and manage HIT. Hematology Am Soc Hematol Educ Program 2011;2011:143-9.

12. Lo GK, Juhl D, Warkentin TE, Sigouin CS, Eichler P, Greinacher A. Evaluation of pretest clinical score (4 T's) for the diagnosis of heparin-induced thrombocytopenia in two clinical settings. J Thromb Haemost 2006;4:759-65.

13. DeBois WJ, Liu J, Lee LY, et al. Diagnosis and treatment of heparin-induced thrombocytopenia. Perfusion 2003;18:47-53. 